\title{
SOME NONLINEAR STOCHASTIC GROWTH MODELS
}

\section{BY HARRY KESTEN}

\begin{abstract}
Stochastic growth models which generalize GaltonWatson branching processes are discussed. The models have an interpretation in population dynamics and economics. The individuals or particles $d o$ interact, e.g. if the individuals represent members of a population, allowance is made for sexual reproduction, so that pairs of individuals are needed to produce offspring. The typical form of the results is that with probability one, either the population remains bounded in size, or it grows at an exponential rate and its composition converges to a fixed point of a suitable transformation.
\end{abstract}

1. Introduction. The problem to which we want to address ourselves is "What is the rate of the population explosion?" In order to deal with this problem mathematically one needs a more or less explicit model for population growth, and we shall consider some probabilistic ones. Stochastic models describing the growth and/or extinction of various populations abound and we only list some of the (historically) more important references and some books or survey articles in which the reader can find further references: Fisher (1930) (Chapter IV has one of the earliest biological applications of branching processes), Feller (1939) (first mathematical treatment of fairly general birth and death processes), Feller (1950) (cf. especially his comments in $\S \S 1,6$ and 7 on the difficulty of treating size and composition of a population with several interacting types), Bartlett (1949) (use of generating functions to handle birth and death processes), Bartlett (1960), Kendall (1949) (cf. especially $\$ 2(\mathrm{ix})$, where the problem of two sexes is discussed), Goodman (1953) and (1968) (considers two sexes with age specific birth and death rates, but has no interactions between the sexes in his stochastic models), Bailey (1957) (models for epidemics), Bharucha-Reid (1960) (Chapter 4 summarizes several models and gives many references), Moran (1962) (especially

An invited address delivered to the Seventy-seventh Annual Meeting of the Society in Atlantic City on January 24, 1971.

AMS 1970 subject classifications. Primary 92A15, 60J80; Secondary 90A15, 60J85, $60 \mathrm{~F} 99$.

Key words and phrases. Stochastic growth model, population growth, exponential growth, growth rate, Malthusian parameter, Galton-Watson branching process, Fisher-Wright-Haldane model, transformation for population composition, stable and unstable fixed points, convergence of composition of population to direction of fixed point, extinction. 
Chapters 1, 5 and 9), Daley (1968) (extinction probabilities for simple stochastic models with two interacting sexes); Harris (1963) and Athreya and Ney (1971) give a rather complete survey of the theory and applications of branching processes, and finally, Smith and Wilkinson (1969) and Athreya and Karlin (1970) and (1971) have treated "branching processes with random environments" (in the latter situations individuals do not behave completely independently of each other anymore).

As far as we can determine the novelty of the considerations below lies in the fact that they allow for several types and two sexes which interact and that they keep track of the size and composition of the population simultaneously. The models are stochastic, but it will turn out that by and large the effect of the random fluctuations is in the initial stages of development. Once the population gets large it will start to grow exponentially, essentially in a deterministic fashion. However, one interesting distinction between the deterministic and stochastic version is discussed at the end of $\S 3$. It is a serious gap in our results that they only predict either extinction or exponential growth of the population. It would be more interesting to find results about the behavior of a population before extinction in a model in which the extinction time is very large with high probability (cf. the comments in Bartlett (1960) pp. 24 and 32 on "quasistationarity"). For the time being we put this last problem aside as being too difficult. Before describing the models we point out that they also have interpretations in other than population genetical terms. E.g., Stigum (1971) and Kesten and Stigum (1972) give an application to economics (see also Example (iv) in \$2).

2. Description of model and examples. To provide a suitable background for discussion we recapitulate the properties of one of the simplest stochastic growth models, to wit, the Galton-Watson branching process (see Harris (1963) or Athreya and Ney (1971) for details and applications). One considers a population with nonoverlapping generations, containing individuals of $d$ types. $Z_{n}(i)$ denotes the number of individuals of type $\mathrm{i}$ (i-individuals) in the $n$th generation. It is assumed that the $(n+1)$ st generation is formed from the $n$th generation in the following way: Each individual produces offspring according to a fixed distribution which depends only on the type of the individual. After that the individual dies and is not counted in the next generation. All individuals produce offspring independently of each other and independent of the past history of the process. This independence assumption is the most crucial one of this model. Let 


$$
m_{i, j}=E\{\# \text { of } \mathrm{j} \text {-children produced by an } \mathrm{i} \text {-individual }\}
$$

and

$$
M=\left(m_{i, j}\right)_{1 \unlhd i, j<d} .
$$

It is easy to write down the conditional expectation of $Z_{n+1}(i)$, given $Z_{0}(\cdot), \cdots, Z_{n}(\cdot)$. Indeed, there are $Z_{n}(j)$ individuals of type $\mathrm{j}$ in the $n$th generation, each of which has an expected contribution of $m_{j, i}$ to $Z_{n+1}(i)$. Thus, the expected number of i-individuals in the $(n+1) \mathrm{st}$ generation from j-parents is $Z_{n}(j) m_{j, i}$, and summing over $j$ one finds

$$
E\left\{Z_{n+1}(i) \mid Z_{0}, \cdots, Z_{n}\right\}=\sum_{j=1}^{d} Z_{n}(j) m_{j, i} \text {. }
$$

In matrix notation, with $Z^{\prime}$ denoting the row vector $(Z(1), \cdots$, $Z(d))$, the transpose of $Z,(2.3)$, reads

$$
E\left\{Z_{n+1}^{\prime} \mid Z_{0}, \cdots, Z_{n}\right\}=Z_{n}^{\prime} M
$$

Iteration of (2.4) gives

$$
E\left\{Z_{n+k}^{\prime} \mid Z_{0}, \cdots, Z_{n}\right\}=Z_{n}^{\prime} M^{k} \text {. }
$$

In order to guess limit theorems for this model we first replace (2.5) by its deterministic analogue

$$
Z_{k}^{\prime}=Z_{0}^{\prime} M^{k}
$$

If one makes the simplifying assumption

$$
d<\infty, \quad 0<m_{i, j}<\infty, \text { for all } i, j,
$$

then it is easy to find the asymptotic behavior of $Z_{k}$ from (2.6). The Perron-Frobenius theorem (Karlin (1966), Appendix) tells us that, under condition (2.7), $M$ has a largest positive eigenvalue $\rho$ with corresponding right and left eigenvectors $u$ and $v$ which are strictly positive, i.e.,

(2.8) $M u=\rho u, \quad v^{\prime} M=\rho v^{\prime}, \quad u(i)>0, \quad v(i)>0, \quad 1 \leqq i \leqq d$.

Moreover, if $u$ and $v$ are normalized such that $u^{\prime} v=1$, then

$$
\left|\frac{M^{n}(i, j)}{\rho^{n}}-u(i) v(j)\right| \leqq K_{1} \lambda^{n}
$$

for some $K_{1}<\infty$ and $0 \leqq \lambda<1$ (see proof of Theorem 2.3 in the Appendix of Karlin (1966)). When applied to (2.6) this theorem shows that $Z_{n}$ grows exponentially at rate $\rho$; more precisely ${ }^{1}$

$1|Z|$ stands for $\sum_{i=1}^{d}|Z(i)|$ if $Z$ is a $d$-vector. 


$$
\left|\frac{Z_{n}(j)}{\rho^{n}}-\left(Z_{0}^{\prime} u\right) v(j)\right| \leqq K_{1}\left|Z_{0}\right| \lambda^{n}
$$

Under mild moment assumptions (and exclusion of a singular case when $\rho=1$ ) this result has the following analogue for the stochastic Galton-Watson process (see Kesten and Stigum (1966) or Harris (1963), Chapter II):

(2.11) If $\rho \leqq 1$, then $Z_{n}=0$ eventually.

If $\rho>1$, then $\lim _{n \rightarrow \infty}\left(Z_{n} / \rho^{n}\right)=w v$ a.e. for some random variable $w$ which satisfies

$$
q=P\{w=0\}=P\left\{Z_{n}=0 \text { eventually }\right\}<1 .
$$

( $\rho$ and $v$ are as in (2.8).) Note that (2.11)-(2.13) imply that, with probability one, $Z_{n}$ either vanishes eventually or grows exponentially at a rate greater than one. If $\rho \leqq 1$, extinction is certain. For $\rho<1$ we could have expected this on the basis of the deterministic model, since

$$
E\left\{Z_{n}^{\prime} \mid Z_{0}\right\}=Z_{0}^{\prime} M^{n}=O\left(\rho^{n}\right) \rightarrow 0 \quad(n \rightarrow \infty, \rho<1)
$$

and $\left|Z_{n}\right|$ can only become very small when $Z_{n}$ vanishes. This last statement is not true in more general cases where $Z_{n}$ does not necessarily have positive integer-valued components. In such cases the alternatives will be: Either $\left|Z_{n}\right|$ remains bounded or $Z_{n}$ grows exponentially at a rate greater than one (see for instance Stigum (1971)). Another important remark concerns the asymptotic direction of $Z_{n}$. It follows from (2.12) and (2.13), that in the case where exponential growth occurs, and this has probability $(1-q)>0$ when $\rho>1$, the limiting direction of $\rho^{-n} Z_{n}$ is nonrandom, but equal to the fixed direction of $v$. Only the asymptotic size of $\rho^{-n} Z_{n}$ is random.

We may consider the problem of the asymptotic behavior of $Z_{n}$ in a Galton-Watson process as settled by (2.11)-(2.13). The reason for these simple and precise results lies in the fact that $E\left\{Z_{n+1}^{\prime} \mid Z_{0}, \cdots, Z_{n}\right\}$ is a linear function of $Z_{n}$ (see (2.4)). This linear relationship is, in turn, a reflection of the assumption that individuals reproduce independently of each other. Each individual produces children by itself without cooperation from other individuals. As is well known from the story of the birds and bees this is not the case in many biological populations. A pair of individuals of opposite sex is needed to produce offspring. We try to take this into account in the following model for zygotic selection, which is a stochastic version of one of the most basic models in population genetics. We shall refer to this as the Fisher-Wright-Haldane model. 
See Mulholland and Smith (1959), Karlin (1962/63), Moran (1962), pp. 50-55, Blakley (1964) and Blakley and Dixon (1964) for a treatment of the deterministic version. Note that this literature only discusses a very special choice of the coefficients $f(\cdot \mid \cdot, \cdot)$ and we shall later also make the same restrictions (see (3.24) and (3.25)). In this model individuals are still not distinguished by sex, but it requires a pair of individuals to produce offspring. More complicated models which do incorporate sex distinctions can be formulated along the same lines (see Kesten (1970)). Despite the fact that sex is not distinguished it is mathematically more convenient to think of couples as ordered pairs. We speak of a $(\mathrm{j}, \mathrm{k})$-couple if the first partner is of type $\mathrm{j}$ and the second partner of type $\mathrm{k}$. Again we consider only a case of nonoverlapping generations without age effects. As before $Z_{n}(i)$ is the number of $\mathrm{i}$-individuals in the $n$th generation and we now also introduce

$$
\left|Z_{n}\right|=\sum_{i=1}^{d} Z_{n}(i)=\text { total size of } n \text {th generation }
$$

and

$$
z_{n}(i)=\frac{Z_{n}(i)}{\left|Z_{n}\right|}=\text { proportion of i-individuals in } n \text {th generation. }
$$

In the Fisher-Wright-Haldane model the $(n+1)$ st generation is formed from the $n$th generation in two steps. First the individuals are divided at random into $\left[\left|Z_{n}\right| / 2\right]$ couples (one individual is left over if $\left|Z_{n}\right|$ is odd), i.e., all possible pairings are taken equally likely. Simple properties of random orderings or drawing without replacement (see Feller (1968), §V. 2(c) with $c=-1, d=0$ and Exercise V. 8.20) show that the probability of a given couple being of type $(j, k)$ equals

$$
z_{n}(j) \frac{Z_{n}(k)-\delta_{j, k}}{\left|Z_{n}\right|-1}
$$

Consequently, for $\left|Z_{n}\right| \geqq 2$ and some $|\theta| \leqq 1$,

$$
\begin{aligned}
& E\left\{\# \text { of }(\mathrm{j}, \mathrm{k}) \text {-couples } \mid Z_{0}, \cdots, Z_{n}\right\} \\
& =\left[\frac{\left|Z_{n}\right|}{2}\right] z_{n}(j) \frac{Z_{n}(k)-\delta_{j, k}}{\left|Z_{n}\right|-1}=\frac{\left|Z_{n}\right|}{2} z_{n}(j) z_{n}(k)+\theta .
\end{aligned}
$$

The second step consists of the production of offspring after the couples have been formed. This time we assume that all couples produce offspring independently of each other, and that the distri- 
bution of offspring depends only on the type of the couple. Instead of (2.1) we now introduce

(2.19) $f(i \mid j, k)=E\{\#$ of $\mathrm{i}$-children produced by a (j, k)-couple $\}$.

Arguments analogous to the ones used to derive (2.3) now lead from (2.18) to

$$
E\left\{Z_{n+1}(i) \mid Z_{0}, \cdots, Z_{n}\right\}
$$

$$
=\frac{\left|Z_{n}\right|}{2} \sum_{j, k} f(i \mid j, k) z_{n}(j) z_{n}(k)+\theta_{1} K_{2},
$$

where $K_{2}=\sum_{i, j, k} f(i \mid j, k),\left|\theta_{1}\right| \leqq 1$.

To compare (2.20) with the formula (2.3) for the Galton-Watson process, and also to come to a more general formulation, we introduce the set of $d$-dimensional probability vectors

$$
A=\left\{x=(x(1), \cdots, x(d)): x(i) \geqq 0, \sum_{i=1}^{d} x(i)=1\right\},
$$

and the following two transformations from $A$ into $A$ :

$$
\begin{gathered}
T_{1} x(i)=\frac{\sum_{j=1}^{d} x(j) m_{j, i}}{\sum_{j, l=1}^{d} x(j) m_{j, l}}, \\
T_{2} x(i)=\frac{\sum_{j, k=1}^{d} x(j) x(k) f(i \mid j, k)}{\sum_{j, k, l=1}^{d} x(j) x(k) f(l \mid j, k)} .
\end{gathered}
$$

(2.3) and (2.20) can then be rewritten as

$$
E\left\{Z_{n+1} \mid Z_{0}, \cdots, Z_{n}\right\}=\left\{\left|Z_{n}\right| \sum_{j, l=1}^{d} z_{n}(j) m_{j, l}\right\} T_{1} z_{n},
$$

respectively

$$
\begin{aligned}
E\left\{Z_{n+1} \mid Z_{0}, \cdots, Z_{n}\right\} & \\
& =\left\{\frac{\left|Z_{n}\right|}{2} \sum_{j, k, l} z_{n}(j) z_{n}(k) f(l \mid j, k)\right\} T_{2} z_{n}+\theta_{2} Y_{n},
\end{aligned}
$$

for some $\left|\theta_{2}\right| \leqq 1$ and a vector $Y_{n}$ with $\left|Y_{n}\right| \leqq d K_{2}$. This suggests that 
we consider positive $d$-vector-valued processes $\left\{Z_{n}\right\}_{n \geqq 0}$ for which the conditional distribution of $Z_{n+1}$, given $Z_{0}, \cdots, Z_{n}$, is "centered around"

$$
\tau\left(Z_{n}(\cdot)\right) T z_{n}
$$

for a nice function $\tau:\left(\boldsymbol{R}_{+}\right)^{d} \rightarrow \boldsymbol{R}_{+}{ }^{2}$ and a nice transformation $T: A \rightarrow A$. In the Galton-Watson process we see from (2.24) that we should take

$$
\tau\left(Z_{n}(\cdot)\right)=\left|Z_{n}\right| \sum_{j, l} z_{n}(j) m_{j, l} \text { and } T=T_{1},
$$

whereas in the Fisher-Wright-Haldane model

$$
\tau\left(Z_{n}(\cdot)\right)=\frac{\left|Z_{n}\right|}{2} \sum_{j, k, l} z_{n}(j) z_{n}(k) f(l \mid j, k) \quad \text { and } \quad T=T_{2}
$$

is the choice prescribed by (2.25).

In the situation described at the end of the last paragraph we look for conditions on $\tau, T$ and the probability mechanism which allow us to conclude that, for some $\rho>1, \rho^{-n} Z_{n}$ converges with positive probability. Firstly, notice that (2.26) is a sort of polar form; the direction of this vector is that of $T z_{n}$, whereas

$$
\left|\tau\left(Z_{n}(\cdot)\right) T z_{n}\right|=\tau\left(Z_{n}(\cdot)\right) .
$$

Thus, if we want to avoid zero or infinite growth rates, i.e., if we want $\left|Z_{n+1}\right|$ to be of the same order as $\left|Z_{n}\right|$, then it seems reasonable to require

$$
K_{3} \leqq \frac{\tau(Z)}{|Z|} \leqq K_{4}, \quad 0 \neq Z \in\left(R_{+}\right)^{d},
$$

for some $0<K_{3} \leqq K_{4}<\infty$. (2.30) holds for (2.27) under the condition (2.7), respectively for (2.28) under mild positivity conditions on $f(\cdot \mid \cdot, \cdot)$. Secondly we want to make precise the condition that $Z_{n+1}$ be "centered around" (2.26). In the Galton-Watson model and the Fisher-Wright-Haldane model we can do this with the help of Chebychev's inequality if we assume that the number of children of each individual, respectively couple, has finite variance. Simple estimates of the conditional variance

$$
E\left\{\left|Z_{n+1}-E\left(Z_{n+1} \mid Z_{0}, \cdots, Z_{n}\right)\right|^{2} \mid Z_{0}, \cdots, Z_{n}\right\}
$$

show that in these cases

$$
{ }^{2} R_{+}=[0, \infty) \text {. }
$$




$$
P\left\{\left|Z_{n+1}-\tau\left(Z_{n}(\cdot)\right) T z_{n}\right| \geqq \alpha\left|Z_{n}\right|^{1-2 \delta} \mid Z_{0}, \cdots, Z_{n}\right\} \leqq K_{5} \alpha^{-2}
$$

for some $K_{5}<\infty, \alpha \geqq 0,\left|Z_{n}\right|>0$ and $\delta=\frac{1}{4}$. In the more general case we shall therefore assume that (2.31) holds for some $0<\delta \leqq \frac{1}{2},\left|Z_{n}\right|>0$ and all $0 \leqq \alpha \leqq K_{6}\left|Z_{n}\right|^{2 \delta}$.

Before we analyze where these assumptions lead us, we want to mention some further examples which fit in the above framework and satisfy (2.30) and (2.31), in order to convince the reader of the value of this set up.

(i) $T_{1}$ and $T_{2}$ are fractional transformations with numerator and denominator homogeneous of degree one, respectively two. However, many models in population genetics lead to more complicated $T$ 's. E.g., Prout (1968), Karlin and Feldman (1968a and b), and Scudo and Karlin (1969), Karlin and Scudo (1969) have investigated mating rules which take into account preferences or antipreferences for mating between individuals of the same type. For several of their rules one can construct a stochastic version analogous to the stochastic version of the Fisher-Wright-Haldane model. (2.30) and (2.31) will again be satisfied but with more complicated $\tau$ and $T$ than in (2.22)(2.28). For the rules of Karlin and Scudo, $T$ of ten is a fractional transformation of degree 3 or 4 . One possible stochastic version of Prout's "polygamous" rule leads to

$$
T x(i)=\frac{\sum_{j, k} f(i \mid j, k) x(j) x(k)\left[\sum_{l} x(l) w(j, l)\right]^{-1}}{\sum_{m, j, k} f(m \mid j, k) x(j) x(k)\left[\sum_{l} x(l) w(j, l)\right]^{-1}}
$$

for suitable $f(i \mid j, k)$ and $w(i, j) \geqq 0$. One is also led to more complicated $T$ 's when one distinguishes individuals by sex (see Kesten (1970)). We remark that complication of $T$ causes major difficulties, whereas complications in $\tau$ are relatively easy to handle.

(ii) Feller (1950), \$10, suggested a model for a population with 2 types. Formula (10.3) of Feller gives the transition probabilities from one generation to the next. In our notation it reads

$$
\begin{aligned}
P\left\{Z_{n+1}(i)=\right. & \left.k_{i}, i=1,2 \mid Z_{0}, \cdots, Z_{n}, Z_{n}(i)=j_{i}, i=1,2\right\} \\
& =\frac{N !}{k_{1} ! k_{2} !\left(N-k_{1}-k_{2}\right) !} p_{1}^{k_{1}} p_{2}^{k_{2}}\left(1-p_{1}-p_{2}\right)^{N-k_{1}-k_{2}},
\end{aligned}
$$

where

$$
N=\sigma_{1} j_{1}+\sigma_{2} j_{2} \quad \text { for some positive integers } \sigma_{1} \text { and } \sigma_{2} \text {, and }
$$




$$
\begin{aligned}
& p_{1}=\frac{\sigma_{1} j_{1}}{N}\left(1-\nu_{1}\right)\left(1-\alpha_{1}\right)+\frac{\sigma_{2} j_{2}}{N}\left(1-\nu_{2}\right) \alpha_{2}, \\
& p_{2}=\frac{\sigma_{1} j_{1}}{N}\left(1-\nu_{1}\right) \alpha_{1}+\frac{\sigma_{2} j_{2}}{N}\left(1-\nu_{2}\right)\left(1-\alpha_{2}\right),
\end{aligned}
$$

for some $0 \leqq \alpha_{i}, \nu_{i} \leqq 1$. One easily derives from properties of the trinomial distribution (2.32) that in this model (2.30) and (2.31) hold with $\delta=\frac{1}{4}$,

$$
\begin{aligned}
T x(1) & =\frac{p_{1}}{p_{1}+p_{2}}=\frac{x(1) \sigma_{1}\left(1-\nu_{1}\right)\left(1-\alpha_{1}\right)+x(2) \sigma_{2}\left(1-\nu_{2}\right) \alpha_{2}}{x(1) \sigma_{1}\left(1-\nu_{1}\right)+x(2) \sigma_{2}\left(1-\nu_{2}\right)}, \\
T x(2) & =1-T x(1)
\end{aligned}
$$

and

$$
\tau(Z)=\sigma_{1}\left(1-\nu_{1}\right) Z(1)+\sigma_{2}\left(1-\nu_{2}\right) Z(2) .
$$

Thus, even though this process is not at all a Galton-Watson process, we are led to a transformation which has the same form as $T_{1}$, which originally came from a Galton-Watson model. Feller did not derive any limit laws for this model, but it follows from our theory that this model will satisfy the limit laws of a Galton-Watson process, i.e., (2.11)(2.13), when one takes

$$
M=\left(\begin{array}{cc}
\sigma_{1}\left(1-\nu_{1}\right)\left(1-\alpha_{1}\right) & \sigma_{1}\left(1-\nu_{1}\right) \alpha_{1} \\
\sigma_{2}\left(1-\nu_{2}\right) \alpha_{2} & \sigma_{2}\left(1-\nu_{2}\right)\left(1-\alpha_{2}\right)
\end{array}\right)
$$

and assumes $0<m_{i, j}<\infty$.

(iii) Professor P. Ney (private communication) has considered some models in which individuals or particles of different types can neutralize each other. It is conceivable that such phenomena occur when an antibody interacts with a tumor cell. Both may be considered inactive after they meet and the model keeps track only of the particles which are still "active". The simplest such model would have only two kinds of particles. If

$$
Z_{n}(i)=\{\# \text { of active particles of type } \mathrm{i} \text { in } n \mathrm{th} \text { generation }
$$

then always $Z_{n}(1) Z_{n}(2)=0$, i.e., only particles of one type remain active after the neutralization takes place. Assume for instance that $Z_{n}(1)>0, Z_{n}(2)=0$. Then the $Z_{n}(1)$ particles of type one produce particles of both types independently of each other and according to a fixed distribution $F_{1}$. The particles themselves die afterwards. Assume $Y_{n}(i)$ particles of type $i, i=1$ or 2 , have been produced. Then neutralization will take place between as many pairs as possible, i.e., between 
$\min \left(Y_{n}(1), Y_{n}(2)\right),(1,2)$-pairs and the $(n+1)$ st generation consists of the remaining particles, i.e.,

$$
Z_{n+1}(i)=Y_{n}(i)-\min \left(Y_{n}(1), Y_{n}(2)\right) .
$$

An analogous description applies when $Z_{n}(1)=0, Z_{n}(2)>0$. In our notation we take $d=2$; at all times one of 3 possibilities occurs: Either $Z_{n}(1)=Z_{n}(2)=0$ or $Z_{n}(1)>0, Z_{n}(2)=0$ or $Z_{n}(1)=0, Z_{n}(2)>0$. Again put

$$
m_{i, j}=E\{\# \text { of } \mathrm{j} \text {-particles produced by an i-particle }\}
$$

and assume that the variance of the number of particles produced by any particle is finite. Assume also

$$
m_{1,1}>m_{1,2} \text { and } m_{2,2}>m_{2,1} \text {. }
$$

Then it follows from Chebychev's inequality that for $\alpha \leqq K_{6}\left\{Z_{n}(1)\right\}^{1 / 2}$

$$
\begin{aligned}
& P\left\{\left|Z_{n+1}(1)-\left(m_{1,1}-m_{1,2}\right) Z_{n}(1)\right|\right. \\
& \left.\geqq \alpha\left\{Z_{n}(1)\right\}^{1 / 2} \text { or } Z_{n+1}(2) \neq 0 \mid Z_{0}, \cdots, Z_{n}\right\} \leqq K_{5} \alpha^{-2},
\end{aligned}
$$

on the set $Z_{n}(1)>0, Z_{n}(2)=0$, and a similar relation holds on $Z_{n}(1)$ $=0, Z_{n}(2)>0$. This again gives (2.30) and (2.31) with $\delta=\frac{1}{4}$ if we take

$$
T(1,0)=(1,0), \quad T(0,1)=(0,1)
$$

and

$$
\begin{array}{ll}
\tau(Z)=\left(m_{1,1}-m_{1,2}\right) Z(1) & \text { when } Z(1)>0, \quad Z(2)=0, \\
\tau(Z)=\left(m_{2,2}-m_{2,1}\right) Z(2) & \text { when } Z(1)=0, \quad Z(2)>0 .
\end{array}
$$

Since $z_{n}=(1,0)$ or $(0,1)$ for all $n$ with $Z_{n} \neq 0$ it is not necessary to define $T$ or $\tau$ elsewhere in this case. Note that another $T$ has to be used when one or both inequalities in (2.33) are reversed.

(iv) A considerable amount of work in mathematical economics is based on the so-called Solow-Samuelson model (Solow and Samuelson (1953)). Here $Z_{n}(i)$ denotes the amount of the $i$ th commodity at time $n$. In the deterministic version it is assumed that

$$
Z_{n+1}(i)=H_{i}\left(Z_{n}(\cdot)\right), \quad 1 \leqq i \leqq d,
$$

where, for $1 \leqq i \leqq d$,

$$
H_{i} \geqq 0, H_{i}(\cdot) \text { is continuous and homogeneous of degree one, }
$$

and

$$
H_{i}\left(Z^{\prime}\right) \geqq H_{i}\left(Z^{\prime \prime}\right) \text { whenever } Z^{\prime}(j) \geqq Z^{\prime \prime}(j), 1 \leqq j \leqq d
$$


For instance $H_{i}$ could be given by

$$
H_{i}(Z)=\prod_{j=1}^{d}\{Z(j)\} \alpha_{i} \quad \text { for some } \alpha_{j} \geqq 0, \quad \sum_{j=1}^{d} \alpha_{j}=1 .
$$

Let $H(Z)$ stand for the vector $\left(H_{1}(Z), \cdots, H_{d}(Z)\right)$ and put

$$
T_{3} z=\frac{H(z)}{|H(z)|}, \quad z \in A, \quad \text { and } \quad \tau(Z(\cdot))=|H(Z)| .
$$

Then, (2.34) can be written in the form

$$
Z_{n+1}=\tau\left(Z_{n}(\cdot)\right) T z_{n} .
$$

Stigum (1971) has considered a stochastic version of this model. He postulated

and

$$
E\left\{Z_{n+1} \mid Z_{0}, \cdots, Z_{n}\right\}=\tau\left(Z_{n}(\cdot)\right) T z_{n}
$$

$$
\sigma^{2}\left\{Z_{n+1}(i) \mid Z_{0}, \cdots, Z_{n}\right\} \leqq K_{7}\left|Z_{n}\right|^{2-2 \delta}
$$

for some $\delta>0, K_{7}<\infty$, instead of the deterministic relation (2.38). By Chebychev's inequality this again gives (2.31) so that Stigum's modification under uncertainty of the Solow-Samuelson model provides another example for our set up (for further work along these lines see Kesten and Stigum (1972)). Note that $T$ can be very complicated in this example (e.g. if (2.37) holds for some $i$ ). However, the assumptions (2.35) and (2.36) make this case quite manageable. In fact an analogue of the Perron-Frobenius theorem was proved for such $T$ by Solow and Samuelson (1953) and Stigum (1971): If the map $Z \rightarrow H(Z)$ is indecomposable, then there exists a unique $\rho>0$ and a unique $v \in A$ such that $H(v)=\rho v$. Moreover all $v(i)>0$. If, for all $1 \leqq i, j \leqq d$,

$$
\left(\frac{\partial H_{i}(Z)}{\partial Z(j)}\right)_{Z=v}
$$

exists and is strictly positive then

$$
\left|\frac{H^{n}(Z)}{\rho^{n}}-\gamma(Z) v\right| \leqq K_{8} \lambda^{n}, \quad n \geqq 0, \quad Z(i)>0,1 \leqq i \leqq d,
$$

for some $\gamma(Z)>0, K_{8}<\infty$ and $0 \leqq \lambda<1$. ( $H^{n}$ is the $n$th iterate of $H$; indecomposability of $H$ is defined in Stigum (1971) and is an analogue of the condition $m_{i, j}>0$ in (2.7). For generalizations to decomposable $H$ see Kesten and Stigum (1972).) As we shall see in the next section this lemma allows one to derive analogues of (2.11)-(2.13). Un- 
fortunately (2.35)-(2.36) do not hold in the Fisher-Wright-Haldane model or other population genetics models. Most importantly, the monotonicity property $(2.36)$ fails; the $(n+1)$ st generation can actually be decreased when the $n$th generation is increased. Indeed one has reduced certain pest populations by releasing large numbers of sterile individuals (see for instance Baumhofer, et al. (1955)).

3. Limit theorems. The first problem we face is to find the candidates for the growth rate or Malthusian parameter $\rho$. We take our cue from a deterministic version of the model. I.e., we consider for a moment the relation

$$
Z_{n+1}=\tau\left(Z_{n}(\cdot)\right) T z_{n}
$$

as strictly valid. In view of (2.29), (3.1) implies

$$
\left|Z_{n+1}\right|=\tau\left(Z_{n}(\cdot)\right), \quad \frac{\left|Z_{n+1}\right|}{\left|Z_{n}\right|}=\frac{\tau\left(Z_{n}(\cdot)\right)}{\left|Z_{n}\right|}
$$

and

$$
z_{n+1}=T z_{n} .
$$

Thus, if $\rho^{-n}\left|Z_{n}\right|$ is to have a positive limit, we must have

$$
\frac{\left|Z_{n+1}\right|}{\left|Z_{n}\right|}=\frac{\tau\left(Z_{n}(\cdot)\right)}{\left|Z_{n}\right|} \rightarrow \rho .
$$

Looking at our examples (2.27) and (2.28) for $\tau$, we see that (3.4) essentially can occur only when $z_{n}$ itself converges and when $\tau\left(Z_{n}(\cdot)\right) /\left|Z_{n}\right|$ is "smooth." Specifically we shall assume that $\tau$ satisfies the following smoothness condition:

There exists a function $\rho: A \rightarrow R_{+}$and constants $K_{9}<\infty$ and $\beta>0$ such that for $x \in A, Z \in\left(\boldsymbol{R}_{+}\right)^{d}$,

$$
\left|\frac{\tau(Z(\cdot))}{|Z|}-\rho(x)\right| \leqq K_{9}\left\{\left|\frac{Z(\cdot)}{|Z|}-x\right|^{\beta}+|Z|^{-\beta}\right\} .
$$

For technical reasons we also have to introduce a Lipschitz condition for $T$ :

$$
|T x-T y| \leqq K_{10}|x-y|, \quad x, y \in A,
$$

for suitable $K_{10}<\infty$. Clearly (3.6) holds for $T_{1}$ and $T_{2}$ and (3.5) for (2.27) and (2.28).

In particular (3.6) requires $T$ to be continuous and therefore $z_{n} \rightarrow p \in A$ is possible only if $p$ is a fixed point of $T$ (see (3.3)). These 
considerations pretty much tell us what to expect in the case where $T$ has a unique fixed point $p \in A$ which is globally strongly stable, in the sense that for some $K_{11}<\infty, 0 \leqq \lambda_{1}<1$,

$$
\left|T^{n} x-p\right| \leqq K_{11} \lambda_{1}^{n} \text {. }
$$

For, in the deterministic case we then have

$$
\left|z_{n}-p\right|=\left|T^{n} z_{0}-p\right| \leqq K_{11} \lambda_{1}^{n},
$$

and if

$$
\rho=\rho(p)>1,
$$

then for sufficiently large $\left|Z_{0}\right|$ we have eventually (by (3.8) and (3.5))

$$
\frac{\left|Z_{n+1}\right|}{\left|Z_{n}\right|}=\frac{\tau\left(Z_{n}(\cdot)\right)}{\left|Z_{n}\right|} \approx \rho>1 .
$$

It is not hard to derive from (3.8), (2.30), (3.5) and (3.9) that

$$
\lim _{n \rightarrow \infty} \frac{Z_{n}(\cdot)}{\rho^{n}}=w p
$$

for some

$$
w=w\left(Z_{0}(\cdot)\right)>0 \quad \text { for }\left|Z_{0}\right| \text { sufficiently large. }
$$

On the other hand, if (3.9) is replaced by

$$
\rho=\rho(p)<1,
$$

then (2.30), (3.8) and (3.5) imply that $\left|Z_{n}\right|$ remains bounded.

If $p$ is not the unique fixed point of $T$, (3.8) may still hold for some fixed point $p$ of $T$. Of course it will now depend on the starting point $z_{0}$ to which fixed point $p z_{n}$ converges. Nevertheless, when $z_{n}$ satisfies (3.8), and (2.30), (3.5) and (3.9) (or (3.13)) hold, then (3.11) (respectively the boundedness of $\left.\left|Z_{n}\right|\right)$ follows. Notice that $\rho$ in (3.11) and (3.13) depends on $p$ so that in this case the eventual grow th rate may depend on the starting point $z_{0}$, and, for certain $z_{0},\left|Z_{n}\right|$ may remain bounded.

Finally we turn to the results for the stochastic model. The first result is an immediate analogue of the above deterministic considerations.

Theorem 1 (Kesten and Stigum (1972)). Let $p$ be a fixed point of the transformation $T: A \rightarrow A$, and $U \subset A$ a neighborhood of $p$ such that 


$$
\left|T^{n} x-p\right| \leqq K_{11} \lambda_{1}^{n}
$$

for some $K_{11}<\infty, 0 \leqq \lambda_{1}<1$, and all $x \in U$. Assume in addition that (2.30), (3.5) and (3.6) hold, as well as (2.31) for $0 \leqq \alpha \leqq K_{6}\left|Z_{n}\right|{ }^{2 \delta}$ for some $K_{6}<\infty$ and $0<\delta \leqq \frac{1}{2}$. Finally assume

$$
\rho(p)>1 .
$$

There then exists a $K_{12}<\infty$ such that

$$
\begin{aligned}
P\left\{\lim _{n \rightarrow \infty} \frac{Z_{n}(\cdot)}{\rho^{n}}=w p(\cdot) \text { for some } w\right. & \left.>0 \mid Z_{0}(\cdot)\right\} \\
& \geqq 1-\frac{K_{12}}{\left|Z_{0}\right|^{2 \delta}} \quad \text { whenever } z_{0} \in U .
\end{aligned}
$$

If instead of (3.15) one has

$$
\rho(p)<1
$$

(but the other conditions still hold) then

$$
P\left\{\left|Z_{n}\right| \rightarrow \infty \text { and } z_{n} \rightarrow p\right\}=0 .
$$

When we compare this theorem with the deterministic results above, we see that the basic content of this theorem is that the stochastic variations do not matter very much once the population becomes large. When $p$ is locally strongly stable (i.e., satisfies (3.14)) and $\rho(p)>1$ then for large $\left|Z_{0}\right|$ the asymptotic direction of $Z_{n}(\cdot)$ will, with high probability, be the direction of $p$, as in (3.11). Also the exponential growth rate is, with high probability, the same as in the deterministic case and the stochastic variations only influence the asymptotic size of $Z_{n}$ through the random factor $w$. The idea of the proof is of course that when $\left|Z_{n}\right|$ is large, then (2.31) guarantees that, with high probability, one has

$$
\frac{\left|Z_{n+1}\right|}{\left|Z_{n}\right|} \approx \frac{\tau\left(Z_{n}(\cdot)\right)}{\left|Z_{n}\right|} \approx \rho\left(z_{n}\right) \text { and } z_{n+1} \approx T z_{n}
$$

By iteration we find that, with high probability,

$$
\frac{\left|Z_{n+k}\right|}{\left|Z_{n}\right|} \approx\left\{\rho\left(z_{n}\right)\right\}^{k}, \quad z_{n+k} \approx T^{k} z_{n} .
$$

But, when also $z_{n} \in U$, then $T^{k} z_{n}$ is within $K_{11} \lambda_{1}^{k}$ of $p$ and the main point is therefore to make sure that $\left|z_{n+k}-T^{k} z_{n}\right|$ is small even when $k$ 
goes to infinity with $n$. (3.6) is just the condition which makes this possible.

Theorem 1 is quite satisfactory when

$T$ has a unique fixed point $p$, which is globally strongly stable,

i.e., when we can take $U=A$ in (3.14). It is then possible to strengthen the conclusion (3.16), in the case $\rho=\rho(p)>1$, to

$$
P\left\{\lim _{n \rightarrow \infty} \frac{Z_{n}}{\rho^{n}}=w p \text { for some } w>0 \text { or }\left|Z_{n}\right| \text { remains bounded }\right\}=1 \text {. }
$$

Also, if $\rho=\rho(p)<1$ and $\left|Z_{n}\right|<1$ implies $Z_{m}=0$ for all $m \geqq n$, as is the case in biological situations, (3.18) can under mild additional conditions be streng thened to

$$
P\left\{Z_{n}=0 \text { eventually }\right\}=1 .
$$

(See Kesten (1970), especially Lemma 6.2, or Kesten (1972).) Admittedly (3.19) is a severe limitation on $T$, but as we saw in $\$ 2$ it is satisfied for the Galton-Watson process $\left(T=T_{1}\right.$, see (2.9)) and the Solow-Samuelson model $\left(T=T_{3}\right.$, see (2.39)). There are, however, still quite a number of problems open when $T$ has more than one fixed point. To avoid serious difficulties we have restricted ourselves to continuously differentiable transformations $T$ with a finite number of fixed points, $p_{1}, \cdots, p_{k}$ say, and such that, for each $x \in A$,

$$
\lim _{n \rightarrow \infty} T^{n} x \text { exists and equals some } p_{i} \text {. }
$$

The ideal theorem for this situation would have as its conclusion

$$
\begin{aligned}
& P\left\{\text { either } \lim _{n \rightarrow \infty} \frac{Z_{n}}{\left(\rho\left(p_{i}\right)\right)^{n}}=w p_{i} \text { for some } w>0\right. \text { and } \\
& \left.\quad \text { some } p_{i} \text { with } \rho\left(p_{i}\right)>1 \text { or }\left|Z_{n}\right| \text { remains bounded }\right\}=1 .
\end{aligned}
$$

We are still far from proving (3.23) in general, but we can prove it for the classical form of the Fisher-Wright-Haldane model which corresponds to the following choice for $f(\cdot \mid \cdot, \cdot)$ :

$$
f(i \mid j, k)=C w_{j, k}\left(\delta_{i, j}+\delta_{i, k}\right)
$$

for some constant $C$ and

$$
w_{j, k}=w_{k, j} .
$$

For this choice of coefficients we shall use $T_{4}$ instead of $T_{2}$, so that 


$$
T_{4} x(i)=\frac{x(i) \sum_{j=1}^{d} x(j) w_{j, i}}{\sum_{l, j=1}^{d} x(l) x(j) w_{l, j}}, \quad 1 \leqq i \leqq d .
$$

Despite the special character of (3.24) and (3.25), $T_{4}$ has considerable importance in genetics. It describes selection operating at one locus with $d$ alleles, but without mutation, i.e., a $(\mathrm{j}, \mathrm{k})$-pair can only have children of type $\mathrm{j}$ or $\mathrm{k}$. The asymptotic behavior of $T_{4}^{n} x$ has been intensively studied and is almost fully known (see for instance the references for the Fisher-Wright-Haldane model given in \$2). A few further conditions are needed in order to justify the conclusion (3.23) for $T_{4}$. We shall only state the most important conditions and refer the reader to Kesten (1972) for the full details. Firstly, it is known that $T_{4}$ has only finitely many fixed points and satisfies (3.22) when

(3.27) all principal subdeterminants of $W=\left(w_{i, j}\right)_{1 \geqq i, j \geqq d}$ are different from 0. (See Karlin (1962/63), Blakley (1964), Theorems 2, 4 and 6, Mulholland and Smith (1959).) Henceforth we assume (3.27). The $\tau$ of (2.28) for the choice (3.24) and (3.25) of coefficients becomes

$$
\tau\left(Z_{n}(\cdot)\right)=C\left|Z_{n}\right| \sum_{j, k} z_{n}(j) z_{n}(k) w_{j, k}
$$

and therefore $\rho(\cdot)$ should be defined by

$$
\rho(x)=C \sum_{j, k} x(j) x(h) w_{j k} .
$$

Our second assumption is that

$$
\rho(p) \neq 1 \text { for all fixed points } p \text { of } T_{4} \text {. }
$$

Our third assumption concerns the linearization $M_{i}$ of $T_{4}$ near its fixed point $p_{i}$. $M_{i}$ is the differential of $T_{4}$ at $p_{i}$. Here we view $T_{4}$ as a transformation of the $(d-1)$ dimensional space $A$ into itself. Thus $M_{i}$ is a linear transformation from $R^{d-1}$ into $R^{d-1}$ which satisfies

$$
\varphi_{i}(T x)=M_{i}\left(\varphi_{i} x\right)+o\left(\left|x-p_{i}\right|\right) \quad\left(x \rightarrow p_{i}, x \in A\right),
$$

where $\varphi_{i}: A \rightarrow R^{d-1}$ is defined by

$$
\varphi_{i}(x)=\left(x(1)-p_{i}(1), \cdots, x(d-1)-p_{i}(d-1)\right) .
$$

Let $\left|\lambda_{1}^{(i)}\right| \geqq \cdots \geqq\left|\lambda_{d-1}^{(i)}\right|$ be the eigenvalues of $M_{i}$. We then assume (3.33) $\left|\lambda_{j}^{(i)}\right| \neq 1$ for $1 \leqq j \leqq d-1$ and all fixed points $p_{i}$ with $\rho\left(p_{i}\right)>1$. 
THEOREM 2 (Without THE FULL SET OF CONDITIONS). If $Z_{n}$ satisfies (2.31) for $T=T_{4}$ and $\tau$ as in (3.28) and if $W$ is such that (3.27), (3.30) and (3.33) hold, then (3.23) holds.

We can say more about the possible limits $p_{i}$ of $z_{n}$. Not every $p_{i}$ with $\rho\left(p_{i}\right)>1$ can occur and this is perhaps the most interesting difference between the deterministic and stochastic case. Let $p_{i}$ be such that

$$
\left|\lambda_{1}^{(i)}\right| \geqq \cdots \geqq\left|\lambda_{s}^{(i)}\right|>1>\left|\lambda_{s+1}^{(i)}\right| \geqq \cdots \geqq\left|\lambda_{d-1}^{(i)}\right| .
$$

Thus $s$ is the number of eigenvalues of $M_{i}$ which exceed one in absolute value. If $s=0$, then $p_{i}$ is locally strongly stable (see Ostrowski (1966), Theorem 22.1 with proof) and Theorem 1 applies to $p_{i}$. If $s=d-1$ then $p_{i}$ is a point of definite repulsion (see Ostrowski (1966), Theorems 22.2 and 21.1 with proofs) and $T_{4}^{n} x \nrightarrow p_{i}$ for any $x \neq p_{i}$. However, if $1 \leqq s \leqq d-1$ then there exists a $(d-1-s)$ dimensional set $S_{i}$, the so called stable manifold of $T_{4}$ at $p_{i}$, such that

$$
T_{4}^{n} x \rightarrow p_{i}, \quad(n \rightarrow \infty) \text { for all } x \in S_{i},
$$

(see Smale (1963), §3, also Kesten (1972)). Thus, there do exist initial points $x$ such that $T_{4}^{n} x$ converges to $p_{i}$ even though $p_{i}$ is locally not strongly stable, i.e. does not satisfy (3.14) when $\left|\lambda_{1}^{(i)}\right|>1$. This, however, is not possible in the stochastic model, i.e. we have the following:

Addendum to Theorem 2. (Under the hypotheses of Theorem 2). $P\left\{\lim _{n \rightarrow \infty} \frac{Z_{n}}{\left(\rho\left(p_{i}\right)\right)^{n}}=w p_{i}\right.$ for some $w>0$, and some $p_{i}$ with $\rho\left(p_{i}\right)>1$ or $\left|Z_{n}\right|$ remains bounded; if $\left|Z_{n}(j)\right| \rightarrow \infty$ for all $j$, then even $\left|\lambda_{l}^{(i)}\right|<1$ for all $\left.1 \leqq l \leqq d-1\right\}=1$.

This addendum justifies, to some extent, the feeling one has that if $p_{3}$ is not stable, then the random fluctuations will move the sample point off $S_{i}$ and eventually to another fixed point (compare the comments in Blakley (1965), \$5). This is indeed the case, if the random fluctuations are large enough, as made precise in condition (3.34) below. Theorem 3 is a general theorem which excludes exponential growth with convergence to a nonstable fixed point. For simplicity we restrict ourselves to interior fixed points. (For details see Kesten (1972).)

Theorem 3. Let $\left\{Z_{n}\right\}_{n \geqq 0}, T$ and $\tau$ satisfy (2.30), (2.31) with $\delta=\frac{1}{4}$ and (3.5). Let $T$ be continuously differentiable and $p=T p, p(i)>0$, $1 \leqq i \leqq d$, and let $M$ be the linearization of $T$ near $p$ (compare (3.31) and 
(3.32)). Assume $\rho(p) \geqq 1$ and that the eigenvalues $\lambda_{1}, \cdots, \lambda_{d-1}$ of $M$ satisfy

$$
\left|\lambda_{1}\right|>1, \quad\left|\lambda_{i}\right| \neq 1, \quad 2 \leqq i \leqq d-1
$$

Finally, let

$$
H=\left\{x \in R^{d}: \sum_{i=1}^{d} x(i)=0\right\}
$$

and assume that for each open set $V<H$, there exist $\delta(V)>0$ and $K(V)<\infty$ such that

$$
\begin{array}{r}
P\left\{\left|Z_{n}\right|^{1 / 2}\left(z_{n+1}-T z_{n}\right) \in V \mid Z_{0}, \cdots, Z_{n}\right\} \geqq \delta(V) \\
\text { on the set }\left\{\left|Z_{n}\right| \geqq K(V)\right\} .
\end{array}
$$

Then

$$
P\left\{\left|Z_{n}\right| \rightarrow \infty \text { and } z_{n} \rightarrow p\right\}=0 .
$$

We conclude this report with a list of some problems, which are immediately suggested by the above.

(a) What happens near a strongly stable fixed point $p$ with $\rho(p)=1$. None of the above theorems covers this situation?

(b) What happens near a fixed point $p$ which is stable but not strongly so, in the sense that $T^{n} x \rightarrow p(n \rightarrow \infty)$ for all $x$ in a neighborhood $U$ of $p$, but (3.14) does not hold? The largest eigenvalue $\lambda_{1}$ of the linearization of $T$ near $p$ must have absolute value one in this case, and $\left|T^{n} x-p\right|$ may decrease only as a negative power of $n$. This situation actually occurs in several biological cases as one sees from the detailed convergence analysis made by Karlin and Feldman (1968a and b), Scudo and Karlin (1969) and Karlin and Scudo (1969).

(c) What is the analogue of Theorem 2 for the Fisher-WrightHaldane model and $T_{4}$ when $T_{4}$ has infinitely many fixed points, i.e., when (3.27) is violated? We do not even know the full story about the behavior of $T_{4}^{n} x$ in the deterministic case then. (See, however, Theorem 5.1 in Blakley and Dixon (1964).) More generally one would like to treat the Fisher-Wright-Haldane model without specializing the coefficients by (3.24) and (3.25). Presumably one has to find a way to patch together a global result from the local Theorems 1 and 3.

(d) Compute or estimate the probabilities of $\left|Z_{n}\right|$ remaining bounded, of the population becoming extinct, or of $z_{n}$ converging to a specific fixed point. These seem to be the most interesting quantities to the population geneticist. 
(e) Derive results about the transient behavior of populations which have a very large extinction time (see the end of $\$ 1$ ). This will probably require other models than the ones considered here.

\section{REFERENCES}

K. B. Athreya and S. Karlin (1970), Branching processes with random environments, Bull. Amer. Math. Soc. 76 (1970), 865-870.

, (1971), Branching processes with random environments. I, II, (to appear).

K. B. Athreya and P. Ney (1971), Forthcoming book on branching processes.

N. T. J. Bailey (1957), The mathematical theory of epidemics, Hafner, New York, 1957. MR $20 \# 1592$.

M. S. Bartlett (1949), Some evolutionary stochastic processes, J. Roy. Statist. Soc. Ser. B 11 (1949), 211-229. MR 11, 672.

(1960), Stochastic population models in ecology and epidemiology, Methuen's Monographs on Appl. Probability and Statist., Methuen, London; Wiley, New York, 1960. MR 22 \#9323.

A. H. Baumhofer, et al. (1955), Screw worm control through release of sterilized flies, J. Econ. Entomol. 48 (1955), 462-466.

A. T. Bharucha-Reid (1960), Elements of the theory of Markov processes and their applications, McGraw-Hill Series in Probability and Statist., McGraw-Hill, New York, 1960. MR 22 \#3032.

G. R. Blakley (1964), Homogeneous nonnegative symmetric quadratic transformations, Bull. Amer. Math. Soc. 70 (1964), 712-715. MR 33 \#5641.

(1965), Darwinian natural selection acting on populations (preprint).

G. R. Blakley and R. D. Dixon (1964), The sequence of iterates of a nonnegative nonlinear transformation, 1964. (preprint).

D. J. Daley (1968), Extinction conditions for certain bisexual Galton-Watson processes, Z. Wahrscheinlichkeitstheorie und Verw. Gebiete 9 (1968), 315-322. MR 37 \#5945.

W. K. Feller (1939), Die Grundlagen der Volterraschen Theorie des Kampfes ums Dasein in wahrscheinlichkeitstheoretischer Behandlung, Acta Bioth. Ser. 5 (1939), 1140. MR 1, 22.

- (1950), Diffusion processes in genetics, Proc. Second Berkeley Sympos. on Math. Statist. and Probability, Univ. of California Press, Berkeley, 1951, pp. 227246. MR 13, 671.

(1968), An introduction to probability theory and its applications. Vol. 1, 3rd ed., Wiley, New York, 1968. MR 37 \#3604.

R. A. Fisher (1930), The genetical theory of natural selection, Clarendon Press, Oxford, 1930.

L. A. Goodman (1953), Population growth of the sexes, Biometrics 9 (1953), 212225. MR 14, 1105.

(1968), Stochastic models for the population growth of the sexes, Biometrika 55 (1968), 469-487. MR 38 \#6855.

T. E. Harris (1963), The theory of branching processes, Die Grundlehren der math. Wissenschaften, Band 119, Springer-Verlag, Berlin; Prentice-Hall, Englewood Cliffs, N. J., 1963. MR 29 \#664.

S. Karlin (1962/63), Lectures on mathematical biology, Stanford University, Stanford, Calif., 1962/63. 
S. Karlin (1966), A first course in stochastic processes, Academic Press, New York, 1966. MR $34 \# 8466$.

S. Karlin and M. W. Feldman (1968a), Analysis of models with homozygote $X$ heterozygote matings, Genetics 59 (1968), 105-116. (1968), 117-136.

S. Karlin and F. M. Scudo (1969), Assortative mating based on phenotype. II, Genetics 63 (1969), 499-510.

D. G. Kendall (1949), Stochastic processes and population growth, J. Roy. Statist. Soc. Ser. B 11 (1949), 230-264. MR 11, 672.

H. Kesten (1970), Quadratic transformations: A model for population growth, Adv. Appl. Probability 2 (1970), 1-82, 179-228. (1972), Limit theorems for stochastic growth models (to appear).

H. Kesten and B. P. Stigum (1966), A limit theorem for multidimensional Galton-Watson processes, Ann. Math. Statist. 37 (1966), 1211-1223. MR 33 \#6707. - (1972), Balanced growth under uncertainty in decomposable economics (to appear).

P. A. P. Moran (1962), The statistical processes of evolutionary theory, Clarendon Press, Oxford, 1962.

H. P. Mulholland and C. A. B. Smith (1959), An inequality arising in genetical theory, Amer. Math. Monthly 66 (1959), 673-683. MR 22 \#1589.

A. M. Ostrowski (1966), Solution of equations and systems of equations, 2nd ed., Pure and Appl. Math., vol. 9, Academic Press, New York, 1966. MR 35 \#7575.

T. Prout (1968), A model for the study of mating interactions, Dept. of Genetics Report, Aarhus Universitet.

F. M. Scudo and S. Karlin (1969), Assortative mating based on phenotype. I, Genetics 63 (1969), 479-498.

S. Smale (1963), Stable manifolds for differential equations and diffeomorphisms, Ann. Scuola Norm. Sup. Pisa (3) 17 (1963), 97-116. MR 29 \#2818b.

W. L. Smith and W. E. Wilkinson (1969), On branching processes in random environments, Ann. Math. Statist. 40 (1969), 814-827. MR 39 \#7684.

R. M. Solow and P. A. Samuelson (1953), Balanced growth under constant returns to scale, Econometrica 21 (1953), 412-424. MR 15, 49.

B. P. Stigum (1971), Balanced growth under uncertainty, J. Econ. Theory (submitted).

Cornell University, Ithaca, New York 14850 